\title{
A Variational Wavelet-Based Computational Model for the Enhancement of Contrast Perception in Color Images
}

\author{
Edoardo Provenzi \\ Departament de Tecnology of Information and Communications, \\ Universitat Pompeu Fabra, C/Tànger 122-140, 08018, Barcelona, Spain \\ edoardo.provenzi@upf .edu
}

\begin{abstract}
One of the most delicate transformations in color image processing is contrast enhancement due to the fact that artifacts and unnatural colors can appear after the process. Here we propose a variational framework in which contrast enhancement is obtained through the minimization of a suitable energy functional of wavelet coefficients. We will show that this new approach has advantages with respect to the usual spatial techniques sustained by the fact that the wavelet representation is intrinsically local, multiscale and sparse. The computational complexity of the model is $\mathcal{O}(N), N$ being the number of input pixels, and the algorithmic implementation is fast thanks to the fact that the functional minimum can be reached through few iterations of Newton-Raphson's method.
\end{abstract}

Keywords: Wavelets, Variational Principles, Contrast, Color Perception.

\section{Introduction}

Contrast enhancement can help improving the detail visibility in images affected by poor global or local contrast. This problem can be induced by, e.g. wrong camera exposition or aperture settings, back-light conditions, high dynamic range of the scene, and so on. For gray-level, state-of-the-art techniques in contrast enhancement have reached a high quality level and real-time or almost real-time performances. However, when we deal with color images, contrast enhancement is a much more complicated procedure because artifact and unnatural colors can appear after the contrast modification.

Since humans are capable of a high-quality color vision, it is quite natural to design algorithms that take inspiration from the Human Visual System (HVS) features in order to produce an efficient enhancement and avoiding the generation of unnatural colors. The algorithms built in this way are usually called perceptually-inspired and their use can be found in research fields as computational photography, image quality, interior design and robotic vision to cite but a few.

S. Tominaga, R. Schettini, and A. Trémeau (Eds.): CCIW 2013, LNCS 7786, pp. 324-333, 2013.

(C) Springer-Verlag Berlin Heidelberg 2013 
In this paper we analyze the problem of perceptual contrast enhancement with variational techniques from the point of view of wavelet theory. For this purpose we propose a functional of detail coefficients whose minimization induces a local and multiscale improvement of contrast. We will show that the EulerLagrange equations of the functional are implicit non-linear equations which enhance the wavelet detail coefficients of the image. By using Newton-Raphson's method those equations can be quickly solved, ensuring a global computational complexity of $\mathcal{O}(N), N$ being the total number of image pixels. Moreover, the sparsity of the wavelet representation allows the algorithm to be even faster.

For the sake of clarity, it is worthwhile to introduce here the notation that we are going to use throughout the paper. Given a discrete RGB image, we will denote by $\mathfrak{I} \subset \mathbb{Z}^{2}$ its spatial domain and by $x \equiv\left(x_{1}, x_{2}\right)$ and $y \equiv\left(y_{1}, y_{2}\right)$ the coordinates of two arbitrary pixels in $\mathfrak{I}$. We will always consider a normalized dynamic range in $[0,1]$, so that a color image function will be $\boldsymbol{I}: \mathfrak{I} \rightarrow[0,1]^{3}$, $\boldsymbol{I}(x)=\left(I_{R}(x), I_{G}(x), I_{B}(x)\right)$, where $I_{k}(x)$ is the intensity level of the pixel $x \in \mathfrak{I}$ in the chromatic channel $k \in\{R, G, B\}$. We stress that every computation will be performed on the scalar components of the image, thus treating independently each chromatic component as in Retinex-like algorithms [12. Therefore, we will avoid the subscript $k$ and write simply $I(x)$ to denote the intensity of the pixel $x$ in a given chromatic channel.

\section{A Perceptual Contrast Functional in the Wavelet Domain}

In this section we shall motivate our choice for the contrast functional to be minimized in order to obtain a perceptually-inspired contrast enhancement in the wavelet domain.

In [3, the authors proved that there exists only a type of contrast functional that comply with a set of basic phenomenological HVS properties: color constancy, i.e. the ability to perceive colors as (almost) the same independently on the illumination conditions, locality of contrast enhancement, exhibited by wellknown phenomena as e.g. Mach bands or simultaneous contrast, and WeberFechner's law of contrast perception, i.e. the logarithmic response of the HVS to changes of spot light intensity. This functional is the following 1 :

$$
C_{w}(I)=\sum_{x \in \mathfrak{I}} \sum_{y \in \mathfrak{I}} w(x, y) \frac{\min \{I(x), I(y)\}}{\max \{I(x), I(y)\}},
$$

where $w: \mathfrak{I} \times \mathfrak{I} \rightarrow(0,+\infty)$ is a weight function that induces locality. The full details about why this functional complies with the basic HVS features

\footnotetext{
${ }^{1}$ In the quoted paper the definition of $C_{w}$ allows an increasing diffeomorphism $\varphi$ to act on the fraction inside the integral and the case $\varphi \equiv$ id, id being the identity map used here, is studied as a subcase. Since $\varphi$ will not have any prominent role in the present paper, we have omitted its presence since the beginning to simplify the notation as much as possible.
} 
listed above can be found in the quoted paper, here we briefly report why the minimization of $C_{w}$ induces contrast enhancement and how it is related to color constancy. Regarding contrast enhancement, observe that the function $c(I(x), I(y))=\frac{\min \{I(x), I(y)\}}{\max \{I(x), I(y)\}}$ is minimized when the minimum intensity value decreases and the maximum increases, which of course corresponds to a contrast intensification. The relation with color constancy comes from the observation that $c$ is a homogeneous function of degree zero, i.e. $c(\lambda I(x), \lambda I(y))=c(I(x), I(y))$ for all $\lambda \neq 0$; in image formation models $\lambda$ is interpreted as the 'color temperature' of the light source that illuminates a scene, thus the homogeneity property implies that the functional $C_{w}$ is able to automatically discard the color cast induced by a global illuminant, coherently with the HVS property of color constancy.

We now want to show how it is possible to recast the variational framework of [3] into the wavelet domain.

For this purpose, let us start recalling that, following the classical reference book [4, an orthogonal wavelet multi-resolution analysis of an image between two scales $2^{L}$ and $2^{J}, L, J \in \mathbb{Z}, L<J$, is given by three sets of detail coefficients $\left\{d_{j, k}^{H}, d_{j, k}^{V}, d_{j, k}^{D}\right\}_{k \in \mathfrak{I}, j=L, \ldots, J}$, which correspond to the horizontal, vertical and diagonal detail coefficients, respectively, completed by $\left\{a_{J, k}\right\}_{k \in \mathfrak{I}}$, the approximation coefficients at the coarser scale. If the image is in color, then each chromatic channel has its own set of detail and approximation coefficients. The set $\left\{a_{J, k}\right\}_{k \in \mathfrak{I}}$ gives a coarse description of the image at the scale $J$ and it is obtained by convolution between the image and a low pass filter. The set $\left\{d_{j, k}\right\}_{k \in \mathfrak{I}}$ is obtained by convolution between the image and a spatially localized band pass filter, so that the $\left\{d_{j, k}\right\}_{k \in \mathfrak{I}}$ give a measure of local contrast in the image at the scale $2^{j}$.

Our proposal for a perceptual contrast functional in the wavelet domain is

$$
\mathcal{C}_{p_{j},\left\{a_{j, k}\right\}}\left(\left\{d_{j, k}\right\}\right)=\sum_{k \in \mathfrak{I}} p_{j} \frac{a_{j, k}}{d_{j, k}},
$$

where $p_{j}$ are positive coefficients that permits to modulate the strength of contrast enhancement. This definition makes sense if the detail coefficients are different from zero, for this reason we fix a threshold $T_{j}>0$ for each scale and consider only those $d_{j, k}$ satisfying $\left|d_{j, k}\right|>T_{j}$; the other coefficients will be left unchanged.

Thanks to the locality of the wavelet representation, the functional $\mathcal{C}_{p_{j},\left\{a_{j, k}\right\}}$ is intrinsically local and does not need the introduction of any further weighting function, which it is instead essential in the spatial variational framework to localize the computation.

If we keep the approximation coefficients fixed and let the other free to vary, then the minimization of $\frac{a_{j, k}}{d_{j, k}}$ corresponds to the intensification of the detail coefficients and thus of local contrast. Also observe that here the basic contrast variable is $\frac{a_{j, k}}{d_{j, k}}$, which is still a homogeneous function of degree 0 as in the variational framework recalled above.

We cannot determine the enhanced detail coefficients solely by minimizing the functional $\mathcal{C}_{p_{j},\left\{a_{j, k}\right\}}$ because that could lead to an uncontrollable over-enhancement 
of contrast, thus we have to introduce a dispersion control term, $\mathcal{D}_{d_{j, k}^{0}}$, that balances the effect of $\mathcal{C}_{p_{j}}$ with a conservative action that tends to maintain the detail coefficients to their original values $\left\{d_{j, k}^{0}\right\}$. In [3] it has been proven that a suitable choice for the dispersion term to preserve dimensional coherence when the contrast is a homogeneous functional of degree 0 is the entropy dispersion, which in the present problem can be written as:

$$
\mathcal{D}_{d_{j, k}^{0}}\left(\left\{d_{j, k}\right\}\right)=\sum_{k \in \mathcal{I}}\left[d_{j, k}^{0} \log \frac{d_{j, k}^{0}}{d_{j, k}}-\left(d_{j, k}^{0}-d_{j, k}\right)\right] .
$$

We then define the wavelet-based perceptually-inspired contrast-enhancement energy as the sum of the two previous functionals, i.e.

$$
\mathcal{E}_{p_{j},\left\{a_{j, k}\right\}, d_{j, k}^{0}}\left(\left\{d_{j, k}\right\}\right)=\left(\mathcal{C}_{p_{j},\left\{a_{j, k}\right\}}+\mathcal{D}_{d_{j, k}^{0}}\right)\left(\left\{d_{j, k}\right\}\right)
$$

By setting to zero the first variation of this energy we find its Euler-Lagrange equations, as we show in the following proposition.

Proposition 1. The minimization of $\mathcal{E}_{p_{j},\left\{a_{j, k}\right\}, d_{j, k}^{0}}$ gives rise to the following Euler-Lagrange equations for the detail coefficients:

$$
\frac{\partial \mathcal{E}_{p_{j},\left\{a_{j, k}\right\}, d_{j, k}^{0}}}{\partial\left\{d_{j, k}\right\}}=0 \Longrightarrow d_{j, k}=d_{j, k}^{0}+p_{j} \frac{a_{j, k}}{d_{j, k}} .
$$

Proof: the computation of the first variation of $\mathcal{D}_{d_{j, k}^{0}}$ with respect to $\left\{d_{j, k}\right\}$ gives:

$$
\frac{\partial \mathcal{D}_{d_{j, k}^{0}}}{\partial\left\{d_{j, k}\right\}}=1-\frac{d_{j, k}^{0}}{d_{j, k}} .
$$

The first variation of $\mathcal{C}_{p_{j},\left\{a_{j, k}\right\}}$ with respect to $\left\{d_{j, k}\right\}$ gives

$$
\frac{\partial \mathcal{C}_{p_{j},\left\{a_{j, k}\right\}}}{\partial\left\{d_{j, k}\right\}}=-p_{j} \frac{a_{j, k}}{\left(d_{j, k}\right)^{2}} .
$$

Summing (6) and (7) we get

$$
1-\frac{d_{j, k}^{0}}{d_{j, k}}-p_{j} \frac{a_{j, k}}{\left(d_{j, k}\right)^{2}}=0
$$

multiplying by $d_{j, k}$ and simplifying the algebraic expression, we arrive to the result stated in Proposition 1 .

We can now summarize the steps of the variational wavelet-based algorithm for perceptual contrast enhancement as follows: 
1. Consider the three chromatic components of an image independently 2 and use the discrete wavelet transform to obtain a multiresolution analysis of each component over a certain number of scales;

2. Compute, for each scale, the new detail coefficients (horizontal, vertical and diagonal) as prescribed by eq. (5) and substitute the original with these new ones;

3. Apply the inverse wavelet transform to obtain the filtered image. In addition to these steps, we operate a linear stretching of the coarser approximation coefficients $a_{J, k}$ in order to maximize the dynamic range reproduced.

The wavelet algorithm previously described has computational complexity $\mathcal{O}(N)$, $N$ being the number of image pixels, and we implemented it in MATLAB using the 'wavelet toolbox'.

Besides the direct and inverse wavelet transformations, the operation that requires more time is the iterative computation of the enhanced detail coefficients, i.e. the resolution of the implicit equation (5). An efficient way to do that is using Newton-Raphson's method [5], initialized with the original values $d_{j, k}^{0}$. Our algorithm stops when the relative error between two subsequent iterations is smaller than $10^{-3}$ and typically convergence is reached in just two, or at maximum three, iterations. Thanks to the quadratic convergence of Newton-Raphson's algorithm and to the low computational cost of the discrete wavelet transform, the wavelet algorithm is considerably faster than the spatial variational algorithm of [3.

In the next section we shall discuss the effect of parameters on the wavelet algorithm and its performances on natural images.

\section{Tests}

As it was presented above, the wavelet algorithm has 4 different types of parameters: 1$)$ the threshold $T_{j}$ beyond which the wavelet coefficients are considered significantly above 0 at the scale $2^{j} ; 2$ ) the number of scales over which the computation is performed; 3 ) the coefficients $p_{j}, 2^{L} \leq 2^{j} \leq 2^{J}$, that express how much we permit to change the original wavelet detail coefficients in each scale; 4) the mother wavelet $\psi$. In the next subsections we shall discuss how the algorithm performances are influenced by these parameters, but before that we would like to show the efficiency of the wavelet algorithm on three images affected by distinct problems: under-exposure, color cast and over-exposure; as can be seen in Figure1 the wavelet algorithm is able to perform a radiometric adjustment of the non-optimally exposed pictures and to strongly reduce the color cast.

\subsection{The Threshold Parameter $T_{j}$}

In the computational algorithm we have set the threshold parameter to be $T_{j} \equiv$ $\frac{\max _{k \in \mathfrak{I}}\left\{d_{j, k}\right\}}{K}, K>1$, for all the scales $2^{j}$. Of course selecting $K \simeq 1$ we deal

${ }^{2}$ Processing color images by performing operations separately on the three chromatic channels is common in all Retinex-like algorithms, that do not use CIELab or similar spaces for their computation. 

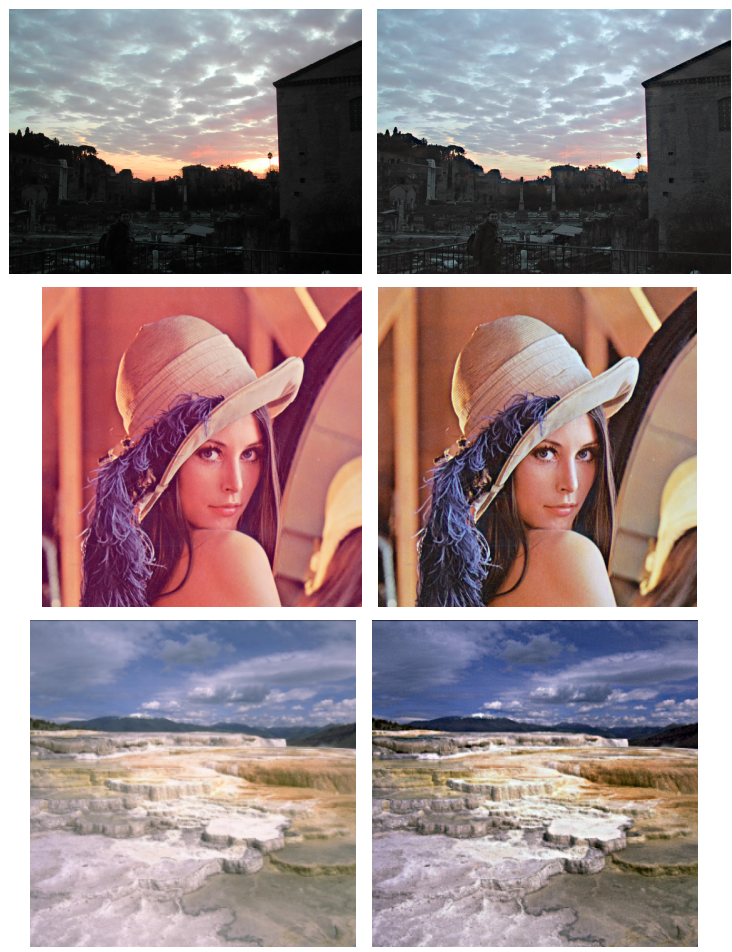

Fig. 1. Images on the left: Original ones. Images on the right: enhanced versions after the wavelet algorithm: details appear in originally underexposed and overexposed areas, and the pink color cast in the 'Lena' image is removed. The filtering parameters are the following: the mother wavelet is the Daubechies wavelet with two vanishing moments, the computation is performed over the maximum number of scales allowed for each image (see Subsection 3.2 for more details), $p_{j}=0.5$, and $T_{j}=\frac{\max _{k \in \mathfrak{I}}\left\{d_{j, k}\right\}}{10}$ for each scale $2^{j}$.

only with the largest detail coefficients, while if we set $K \gg 1$ we introduce in the computation also the smaller ones. Our tests have shown that an optimal value for $K$ is 10 for every scale, in fact, selecting values of $K$ bigger then 10 the algorithm does not introduce significant improvement in detail rendition but it may have the unwanted effect to intensify the noise corresponding to small detail coefficients. Thus, we have set once and for all $T_{j}=\frac{\max _{k \in \mathfrak{I}}\left\{d_{j, k}\right\}}{10}$ for all the scales $2^{j}$, which means that we only deal with the detail coefficients that lie in the same decimal order of magnitude of the biggest ones. In Figure 2 we show the effect of decreasing too much the threshold $T_{j}$.

\subsection{The Number of Scales}

The number of scales $J-L$ that can be used depends on the image dimension and the width $\mathcal{W}_{\psi}$ of the mother wavelet support. In fact, the maximum number of 


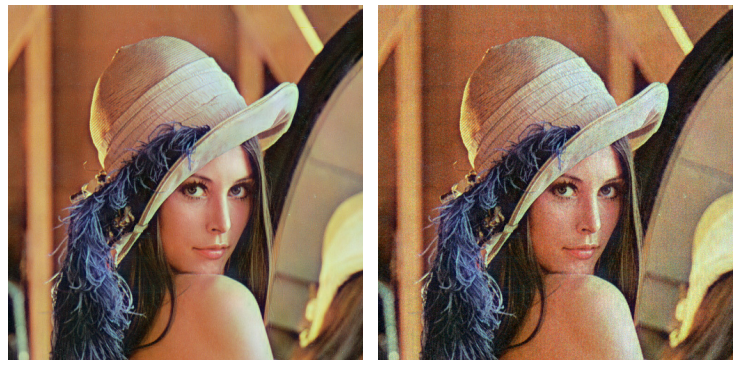

Fig. 2. From left to right: 'Lena' image filtered with the wavelet algorithm with decreasing values of the threshold $T_{j}=\frac{\max _{k \in \mathfrak{I}}\left\{d_{j, k}\right\}}{K}$ corresponding to $K=10$ and 50, respectively. We can see that when $K=50$ the resulting image is affected by noise due to the intensification of small detail coefficients corresponding to noise. The other parameters are maintained fixed: the computation is performed over the maximum number of scales allowed for each image (see Subsection 3.2 for more details), $p_{j}=0.5$ for each scale, and the mother wavelet is 'Sym8', the Symlet with support a with of 15 pixels (arbitrary chosen).

meaningful scales is the highest value of $J-L$ such that the following inequality holds: $2^{J-L} \mathcal{W}_{\psi} \leq \min \{\operatorname{width}(\mathfrak{I})$, height $(\mathfrak{I})\}$. This value can be automatically computed with the command 'wmaxlev' in the MATLAB wavelet toolbox. Our tests have shown that the best contrast enhancement performances of the wavelet algorithm in terms of detail rendition and elimination of color cast corresponds to the highest number of scales allowed. For this reason we have used the command 'wmaxlev' to automatically set the number of scales over which carry on the computation of the enhanced detail coefficients, thus eliminating the variability of this parameter.

\subsection{The Contrast Enhancement Coefficients $\boldsymbol{p}_{\boldsymbol{j}}$}

From eq. (5) it follows that, if we increase the value of the coefficients $p_{j}$, the effect of contrast enhancement becomes more intense. However, if we increase them too much, contrast can be over-enhanced, resulting in unpleasant images with unnaturally high contrast. This effect is shown in Figure 3, where we show the difference produced by increasing the coefficients $p_{j}$ of one order of magnitude.

In general, setting $p_{j}=0.5$ corresponds to overall good performances of the wavelet algorithm, thus 0.5 can be considered as a 'reference value' for the coefficients $p_{j}$. However, since their setting is very intuitive, they can also be easily tuned around this reference value by a user that may want more or less contrast enhancement. 

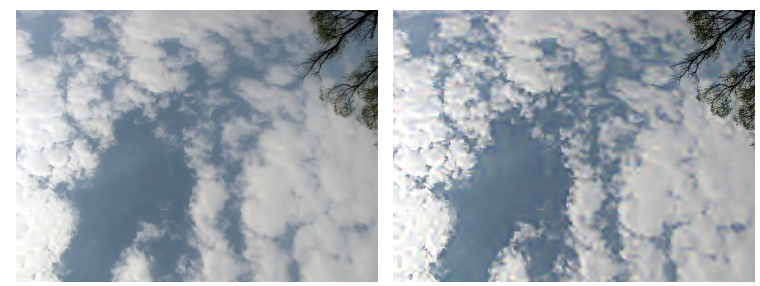

Fig. 3. From left to right: effect of increasing the coefficients $p_{j}$ from 0.5 to 5 , respectively. The images filtered with $p_{j}=10$ have unnatural high contrast. In all the computations the other parameters are maintained fixed: the computation is performed over the maximum number of scales allowed, $T_{j}=\frac{\max _{k \in \mathfrak{I}\{}\left\{d_{j, k}\right\}}{10}$ for each scale and the mother wavelet is the 'Sym8' (arbitrarily chosen).

\subsection{The Mother Wavelet $\psi$}

Different mother wavelets $\psi$ have, in general, different support and symmetry properties 3 . As a consequence, different mother wavelets induce different local contrast enhancement, as can be seen in Figure 4. How to properly choose the family of wavelet is still an open problem that we would like to address in the future.

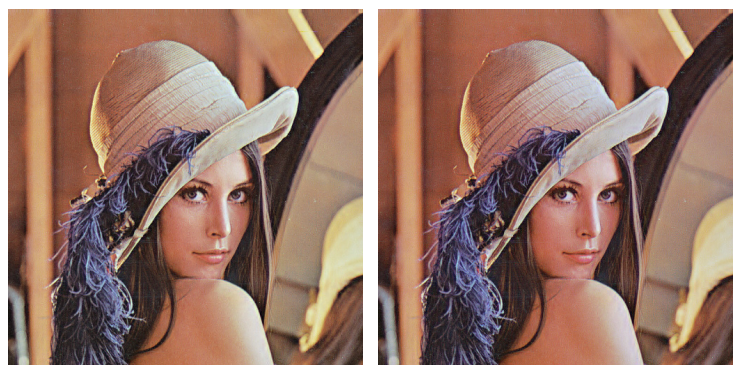

Fig. 4. From left to right: output of the wavelet algorithm obtained with the Daubechies and Coiflet wavelet, respectively, with 4 vanishing moments. The other parameters are maintained fixed: the computation is performed over the maximum number of scales allowed, $T_{j}=\frac{\max _{k \in \mathfrak{I}}\left\{d_{j, k}\right\}}{10}$ and $p_{j}=0.5$ for each scale.

Let us suppose that a give wavelet class is chosen, then one has a further degree of freedom given by the number of vanishing moments. These ones have a strong relation with local contrast: it can be proved (see [4]) that the bigger is the number of vanishing moments of $\psi$, the higher must be the image contrast detected in the support of $\psi$ to get detail coefficients appreciably different from zero. So,

${ }^{3}$ For an extensive discussion about mother wavelet properties, the interested reader is referred to chapter 7 of the standard book 6 . 

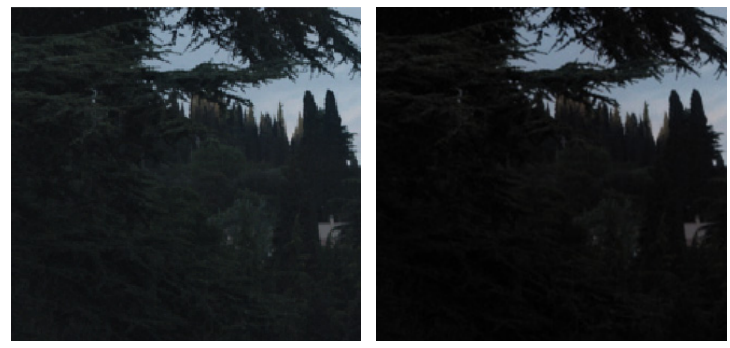

Fig. 5. From left to right: output of the wavelet algorithm obtained with the Daubechies wavelet with 3 and 8 vanishing moments, respectively. The other parameters are maintained fixed: the computation is performed over the maximum number of scales allowed, $T_{j}=\frac{\max _{k \in \mathfrak{I}}\left\{d_{j, k}\right\}}{10}$ and $p_{j}=0.5$ for each scale.

the rationale for choosing the number of moments of a mother wavelet within the wavelet-based algorithm discussed in this paper is the following: if a user is interested in highlighting only high contrast regions, then a wavelet with a high number of vanishing moments should be selected; viceversa, if one is also interested in enhancing lower contrast regions, then a smaller number of vanishing moments must be preferred. This fact is best shown in dark image zones, as in Figure 5, where we show the effect of changing the number of vanishing moments of the Daubechies wavelet from 3 to 8 . Coherently with what stated above, it can be seen that the contrast enhancement on low contrast areas provided by a wavelet with a smaller number of vanishing moments is better since a greater number of detail coefficients appreciably greater than zero can be enhanced.

\section{Conclusions and Perspectives}

We have proposed a variational model of perceptually-inspired contrast enhancement of color images based on the wavelet representation. The wavelet framework allows introducing a new definition of perceptual contrast which permits to construct a fast algorithm that can be used to intensify contrast in color images without introducing artifacts or unnatural colors.

The wavelet algorithm is intrinsically local and has computational complexity $\mathcal{O}(N), N$ being the number of image pixels, and it can be parallelized in order to achieve real-time performances even for large images, thus it could be also used to efficiently process video sequences (e.g. to reduce flickering or remove color cast due to film ageing). This improvement with respect to the variational algorithm presented in [3] is provided by the sparsity of the wavelet representation and by quadratic convergence of Newton-Raphson's algorithm, which is used to solve the implicit equations that give the enhanced detail coefficients.

Qualitative and quantitative tests about the wavelet-based algorithm shows that it is able to enhance both under and over exposed images and to remove color cast, as the spatial variational method of [3]. 
The wavelet framework points out new issues whose discussion is beyond the scope of this paper, but that we consider interesting for future investigation: 1) What is the relation between the intrinsic features of the mother wavelet $\psi$, i.e. shape, support width and symmetry, and the color normalization abilities of the wavelet algorithm? 2) Can we devise an analogue model by suitably apply the windowed Fourier transform to the spatial variational algorithm presented in [3]? If so, how does that model relates to the one described in this paper? 3) Which is the optimal selection of the coefficients $p_{j}$ for contrast enhancement?

4) Can neuroscience models of vision provide insights to properly choose the mother wavelet $\psi$ and the coefficients $p_{j}$ or to guide towards a more complete model?

We are particularly intrigued by this final point since, as discussed in [7], Gabor wavelets can play a major role in answering question 4): we are now performing a theoretical research on the relationship between the model presented in this paper and that of [7]. This could eventually lead us to select once and for all the best suited mother wavelet, thus fixing a tricky parameter of the algorithm presented in this paper.

Acknowledgements. The author acknowledges partial support by PNPGC project, reference MTM2006-14836, and by GRC reference 2009 SGR 773 funded by the Generalitat de Catalunya. He also acknowledges the Ramón y Cajal fellowship by Ministerio de Ciencia y Tecnología de España.

\section{References}

1. Land, E., McCann, J.: Lightness and retinex theory. Journal of the Optical Society of America 61, 1-11 (1971)

2. Rising, H.K.: Analysis and generalization of retinex by recasting the algorithm in wavelets. Journal of Electronic Imaging 13(1), 93-99 (2004)

3. Palma-Amestoy, R., Provenzi, E., Bertalmío, M., Caselles, V.: A perceptually inspired variational framework for color enhancement. IEEE Transactions on Pattern Analysis and Machine Intelligence 31, 458-474 (2009)

4. Mallat, S.: A Wavelet Tour of Signal Processing, 3rd edn. Academic Press (1999)

5. Fausett, L.: Applied Numerical Analysis Using MATLAB. Prentice Hall (2007)

6. Daubechies, I.: Ten Lectures on Wavelets. SIAM (1992)

7. Lee, T.: Image representation using $2 d$ gabor wavelets. IEEE Transactions on Pattern Analysis and Machine Intelligence 18, 1-13 (1996) 